\title{
Roadmap to clinical use of gold nanoparticles for radiosensitization
}

\author{
J. Schuemann ${ }^{1}$, R. Berbeco ${ }^{2}$, D. Chithrani ${ }^{3}$, S. Cho ${ }^{4}$, R. Kumar ${ }^{5,6}$, S. McMahon ${ }^{1,7}$, \\ S. Sridhar ${ }^{5,6}$, and S. Krishnan ${ }^{8}$
}

${ }^{1}$ Department of Radiation Oncology, Massachusetts General Hospital and Harvard Medical School, Boston, MA, USA

2 Department of Radiation Oncology, Brigham and Women's Hospital, DanaFarber Cancer Institute and Harvard Medical School, Boston, MA, USA

${ }^{3}$ Department of Physics, Ryerson University,Toronto, Ontario, Canada

${ }^{4}$ Department of Radiation Physics \& Department of Imaging Physics, The University of Texas MD Anderson Cancer Center, Houston, TX, USA

${ }^{5}$ Nanomedicine Science and Technology Center, Northeastern University, Boston, MA, USA

${ }^{6}$ Department of Radiation Oncology, Harvard Medical School, Boston, MA

${ }^{7}$ Centre for Cancer Research and Cell Biology, Queen's University Belfast, Belfast, Northern Ireland

${ }^{8}$ Department of Radiation Oncology, The University of Texas MD Anderson Cancer Center, Houston, TX, USA

Running title: Roadmap to clinical use of GNPs

\section{Acknowledgements:}

The authors would like to thank Yuting Lin for providing figure 2A.

J.S. acknowledges support in part by the ECOR Formulaic Bridge Funding from the Massachusetts General Hospital and $\mathrm{NIH} / \mathrm{NCI}$ grant R01CA187003.

R.B. acknowledges support in part by $\mathrm{NIH}$ grant R21CA188833 and R03CA164645 from the National Cancer Institute.

S.C. and S.K. acknowledge support in part by NIH grant CA155446, DoD/PCRP grant W81XWH-12-1-0198 and the MD Anderson Cancer Center support grant P30 CA16672.

R.K. and S.S. acknowledge support in part by NSF grant DGE 0965843 and $\mathrm{NIH/NCl}$ grant R03CA164645. 


\begin{abstract}
The past decade has seen a dramatic increase in interest in the use of Gold Nanoparticles (GNPs) as radiation sensitizers for radiotherapy. This interest was initially driven by their strong absorption of ionizing radiation and the resulting ability to increase dose deposited within target volumes even at relatively low concentrations. These early observations are supported by extensive experimental validation, showing GNPs' efficacy at sensitizing tumors in both in vitro and in vivo systems to a range of types of ionizing radiation, including kilovoltage and megavoltage $\mathrm{X}$-rays as well as charged particles.

Despite this experimental validation, there has been limited translation of GNPmediated radiosensitization to a clinical setting. One of the key challenges in this area is the wide range of experimental systems that have been investigated, spanning a range of particle sizes, shapes and preparations. As a result, mechanisms of uptake and radiosensitization have remained difficult to clearly identify. This has proven a significant impediment to the identification of optimal GNP formulations which strike a balance among their radiosensitizing properties, their specificity to the tumors, their biocompatibility, and their imageability in vivo. This white paper reviews the current state of knowledge in each of the areas concerning the use of GNPs as radiosensitizers, and outlines the steps which will be required to advance GNP-enhanced radiation therapy from their current preclinical setting to clinical trials and eventual routine usage.
\end{abstract}




\section{Introduction}

\subsection{History}

High atomic number $(Z)$ elements such as iodine and barium have a long history as imaging contrast agents. Various imaging techniques exploit their high X-ray attenuation coefficients to improve image contrast. As these elements also have much higher energy absorption coefficients than soft tissue, their presence at a disease site will increase the effective dose delivered at the site, opening the potential to use high-Z elements in therapeutic settings (1). Despite this potential, the development of contrast agents for radiotherapy has been hampered by numerous challenges, such as developing an agent with sufficient tumor specificity, which provides good contrast, and which can provide a therapeutic benefit at practical concentrations.

Interest in this field has greatly increased in the last decade, with a particular focus on gold nanoparticles (GNPs). These particles, typically defined as gold structures with the longest dimension smaller than $100 \mathrm{~nm}$, provide excellent radiation contrast due to gold's high atomic number $(Z=79$, see figure 1$)$, are biologically well tolerated and were observed to passively accumulate in tumors while being effectively excluded from healthy tissue. Early work by Hainfeld et al (2) showed their potential as radiosensitizers by demonstrating natural tumor specificity and substantial improvements in tumor control in mice receiving kilovoltage radiotherapy minutes after injection of GNPs. Hainfeld et al's results prompted a range of theoretical and experimental studies of the radiosensitizing effects of GNPs.

Figure 1

Figure 1: Mass energy absorption for gold and soft tissue and the ratio. Figure with permission from (3).

\subsection{Current Status}

To date there have been several hundred articles published on GNP-mediated radiosensitization. There is a broad consensus that GNPs can significantly sensitize cells to ionizing radiation both in vitro (with reported dose enhancement factors on the order of 10 to $100 \%$ ) and in vivo. Moreover, this has been demonstrated for a range of different radiation types, including keV photons from brachytherapy and kilovoltage (kV) sources, megavoltage (MV) photons, MeV electrons and heavy charged particles (2, 4-7), suggesting broad clinical applicability. Importantly, the observed radiosensitization is often significantly greater than the dose enhancement predicted by simple macroscopic dose models, suggesting that more complex physical, chemical or biological interactions are involved in GNP-mediated radiosensitization. 
As a result, while delivering a sufficiently large amount of GNPs to cause macroscopic dose enhancement may prove clinically challenging, effective radiosensitization has been observed with targeted GNPs at much lower concentrations, such as $10 \mu \mathrm{g}$ gold/g body weight in mice (6). These concentrations translate into total injection doses on the order of $1 \mathrm{~g} \mathrm{Au}$ for a single human treatment, levels which are likely to be acceptable with regards to both toxicity and financial considerations. It should be noted that the magnitude of enhancement in radiosensitization observed in experiments at this level is not predicted by current mechanistic analyses. Thus the radiosensitization is most likely a combination of an increase in cell stress through biological effects or some yet to be described enhancement mechanism in combination with the increase in local radiation dose.

However, while achievable, it remains unclear how best to optimize these effects. Experimental studies of GNPs have made use of many different formulations. Variations of size, shape and surface coating all have been shown to significantly impact sensitization, often with conflicting results (3). GNP-mediated radiosensitization also appears to be highly dependent on the system being studied, with varied responses shown both between different cell lines in vitro as well as in different animal model systems.

These uncertainties hamper the development of GNPs towards clinical practice, with several key questions that must be addressed before taking a candidate particle forward into a trial setting. These include: the selection of a site which is expected to see the greatest benefit from the introduction of GNP contrast agents; the development of a particle which offers a balanced design between biodistribution and radiosensitizing properties; the concentration of GNPs necessary to achieve a significant radiosensitization; the validation that such particles can be practically and cost-effectively delivered as part of a fractionated course of radiotherapy; the nature of radiotherapy used; and the influence of concurrent chemotherapy on the effectiveness of GNP-mediated radiosensitization.

This report summarizes the issues involved in advancing GNP-enhanced radiation therapy from the bench to the bedside and lays out a roadmap to potentially address these issues.

\section{Physical effects and modeling of GNP interactions}

Understanding the changes to the radiation field caused by the presence of GNPs is the first step in understanding GNP-mediated radiosensitization. The physics processes involved in GNP enhanced radiation therapy depend on the radiation modality.

\section{$2.1 \mathrm{keV}$ photons}

Originally, GNPs were only expected to produce observable effects at high GNP concentration and with $\mathrm{keV}$ photon treatments. For example, with $7 \mathrm{mg}$ gold $/ \mathrm{g}$ tumor, the dose enhancement was predicted to be as high as $110 \%$ for $140 \mathrm{kVp}$ X-rays (8) and $70 \%$ for $\mathrm{I}-125$ gamma rays (9). Gold's photoelectric cross section for $\mathrm{keV}$ photons is large compared to that of soft tissue especially for photons with energies around the L- and K-shell excitation energies $(\sim 13$ and $\sim 81 \mathrm{keV}$, 
respectively; see figure 1). The emission of inner shell electrons in gold can result in an Auger cascade, which produces around 10-20 low energy (Auger and Coster-Kronig) electrons. While most of these electrons are absorbed inside the GNP, between 1 and 7 electrons typically escape GNPs of 2-50 nm diameter (10). These low energy electrons deposit their energy in close proximity to the GNP, resulting in high dose depositions around the GNPs. The dose deposited by the Auger electrons originating in the GNPs falls off by over $99 \%$ after within the first $250 \mathrm{~nm}$ (see figure 2a). This is independent of the incident radiation type or energy as the energy of Auger electrons is determined by the energies of the electron transitions. However, as seen in figure $2 \mathrm{a}$, even the dose deposit including all electrons generated in the GNPs falls off to below $99 \%$ within 250 $\mathrm{nm}$ (11). The highest energy Auger electrons and photoelectric electrons can deposit dose up to several micrometers away from the GNP. Monte Carlo simulation studies have shown that the microscopic dose enhancement for keV photons can be increased by as much as a factor of 3000 compared to dose originating from a hypothetical water nanoparticle at distances of around $10 \mu \mathrm{m}$ $(11,12)$. Theoretical studies have shown that local doses can be enhanced even more for highly ionized GNPs when irradiated with monoenergetic photons at 68 $\mathrm{keV}(13)$.

\subsection{MV photons}

MV photons are the most commonly used radiation type for cancer treatment. The dominant interaction with gold for MV photon beams is through Compton scattering. The Compton scattering cross section is about 3 orders of magnitude lower than the photoelectric cross section for keV photons, and similar to that of soft tissue. Thus, MV photons were not expected to cause significant GNPmediated radiosensitization. However, MV photon beams contain an increasing percentage of $\mathrm{keV}$ photons and electrons when penetrating a patient or water phantom. In air, the fraction of low energy $(<150 \mathrm{keV})$ photons is about $0.5 \%$. Due to scattering processes, this increases to $13 \%$ at $10 \mathrm{~cm}$ depth or $20 \%$ for a flattening filter free (FFF) beam. Additional modifications of the treatment beam can further increase the low energy photon content $(8,14,15)$. This "beam softening" increases the effectiveness of GNPs. Changing the energy of the electron beam incident on the target can also modify the low energy component. It has been shown that the dose enhancement induced by the secondary electron component of a clinical $6 \mathrm{MV}$ photon beam is in the same order as that from the photon component. The increase in dose from a GNP compared to a hypothetical water nanoparticle is a factor 10 to 100 at distances greater than 1 $\mu \mathrm{m}$ from the GNP surface $(11,12,15)$.

\subsection{Protons}

The dominant process for proton interactions with gold is the production of secondary electrons via small angle scattering due to the higher density of gold as compared to soft tissue $\left(19.3 \mathrm{~g} / \mathrm{cm}^{3}\right.$ vs. $\left.\sim 1 \mathrm{~g} / \mathrm{cm}^{3}\right)$. In contrast to inner shell ionizations seen in photon-gold interactions, these events primarily involve the ejection of low-energy secondary electrons from outer atomic orbitals. 
Consequently, Auger electrons were found to contribute only about $1.5 \%$ of the GNP-mediated dose. Proton irradiation with clinically used energies produces secondary electrons with the lowest energy of the three radiation types discussed here. This results in a comparable dose enhancement to MV photons in close proximity $(\sim 200 \mathrm{~nm})$ to the GNPs but considerably lower effects at distances above $1 \mu \mathrm{m}$ (see figure 2) (11).

Figure 2

Figure 2: Illustration of the radiosensitization effect of GNPs: A) Comparison of the radial dose distribution per ionization as obtained from Monte Carlo simulations performed with TOPAS (16) for a single $50 \mathrm{~nm}$ GNP at the center of a proton spread out Bragg peak (SOBP), for a $250 \mathrm{kVp}$ photon field at $1 \mathrm{~mm}$ depth in water and for the electron and photon components of a Varian $6 \mathrm{MV}$ field at the $80 \%$ depth dose position, B) a $10 \%$ dose enhancement calculated for the addition of GNPs to a uniform 2 Gy by $50 \mathrm{keV} \mathrm{X-ray} \mathrm{irradiation} \mathrm{if} \mathrm{the} \mathrm{additional}$ dose is calculated with macroscopic methods vs. C) the dose enhancement when considering doses at the microscopic scale around individual GNPS, and $D$ ) the resulting RBE caused by the addition of GNPs comparing macroscopic and microscopic (based on an approach following the Local Effect Model) biological modeling for keV $X$-ray irradiation.

\subsection{Modeling of biological effect}

Early models investigated the macroscopic dose enhancement induced by the presence of GNPs (8). Due to discrepancies with experimental observations, more recent studies consider microscopic dose distributions $(9,12,17,18)$. Highly localized energy deposition by GNPs lead to spikes in dose, resembling the dose pattern deposited by radiations with high linear energy transfer such as ions or low energy protons (see figure 2).

In order to estimate the effectiveness of ion therapy, biological effect models are employed that consider these highly localized dose depositions. The same approach can be applied to GNP-mediated dose enhancement. Adaptations of the local effect model (LEM) have been used to translate the microscopic dose enhancement to predict cell survival for various radiation modalities. These models generally predict a much higher sensitizer enhancement ratio than when only considering the macroscopic dose enhancement. Sensitization in LEMbased models depends greatly on the location of GNPs and the radiation modality. In general, the closer GNPs are to the nucleus of target cells, the larger the effect.

It is important to note that these biological calculations are extremely sensitive to dose distributions on the nanometer scale. At these length scales, there are significant uncertainties in the Monte Carlo toolkits often used in these calculations, relating to uncertainty in atomic and molecular cross-sections for 
low-energy processes as well as neglected processes such as free radical formation and transportation. Further developments of these models may prove important to properly understand the physical mechanisms of GNP-enhanced radiation therapy. Monte Carlo simulations provide the currently most accurate platform to model the interactions of radiation with GNPs and the sub-cellular components and can be used to explain and then optimize the radiation type and targeting dependent radiosensitization.

\section{Determinants of Uptake and Radiosensitization in Biological Systems}

Engineering optimal GNPs for radiosensitization requires understanding of the physicochemical interactions of a synthetic nanoparticle with biological systems for the sake of simplicity, this can be pared down to interactions of nanoparticles with individual cells in culture and interactions of nanoparticles with tissues in living subjects.

\subsection{In-vitro uptake and radiosensitization}

\subsubsection{Size, Shape and Concentration}

Due to the increased interaction probability, the GNP concentration has a direct influence on radiosensitization (see figure $3 \mathrm{~A}, \mathrm{~B}$ ); greater concentrations result in more radiosensitization $(19,20)$. GNP concentrations used in those studies were in the range of $1.5 \mathrm{nM}$ and the concentrations used were well tolerated in vitro and had minimal influence on cellular proliferation over a period of two population doubling times. Addition of serum to the medium results in a significant decrease in internalization of untargeted GNPs due to adsorption of serum proteins to the nanoparticle surface and hindrance of particle interaction with cell membranes (20).

The GNP size is an important determinant of intracellular GNP concentration, and thus radiosensitization. Numerous studies have reported on the effects of nanoparticle size on uptake for a variety of particle preparations. These reports agree that there is an optimum diameter in the range of $10-50 \mathrm{~nm}$ (see figure $3 \mathrm{~A}$ ) (21), but the exact value appears to be coating- and cell-line dependent. In vitro clonogenic survival studies have demonstrated a strong correlation with particle uptake (see figure 3B) (22). The purported mechanism of size dependence for internalization is the competing need for thermodynamic (elastic) energy for bending and wrapping of the cell membrane around smaller particles during endocytosis and the need for trafficking receptors from farther away to aid endocytosis of larger particles (23). It was shown that the presence of GNPs causes an increase in DNA double strand breaks during radiation treatment (21) mainly due to the creation of more free radicals to be discussed in the section 3.1.3 (see figure 3D) (24).

The shape of particles also influences the amount of internalization in two ways, first by increasing the overall surface area available to attach targeting ligands, second, by altering the local curvature to fit the contours of cell membranes and thus increasing the surface interaction with ligands and serum proteins (25). 
Figure 3

Figure 3: Size dependence of GNP uptake and sensitization. A) Cellular uptake of GNPs with a diameter size of 10-100 $\mathrm{nm}$. B) Cell survival curves based on a clonogenic assay for the same cells as in A. C) Cells that internalized GNPs showed more DNA double strand breaks after radiation compared to the control cells with no internalized GNPs. D) Schematic showing enhanced free radial formation during the radiation treatment in the presence of GNPs (reprinted with permission) $(20,21,24)$.

\subsubsection{Surface properties}

Efforts to increase the bio-stability of GNPs have largely centered on coating them with a layer of polyethylene glycol (PEG) to provide a hydrophilic layer around the particles which keeps the GNPs from aggregating and enables them to evade macrophage recognition as foreign objects. In vitro, however, PEGylation decreases internalization (20) - saturation of the surface density of PEG and optimal chain lengths of PEG corresponding to a molecular weight of 2000 Daltons resulted in the least uptake by macrophages in an in vitro assay (26). A technique frequently employed to drive internalization of GNPs into cells is to conjugate them with biomolecules that dock to cell surface proteins. By using such a combined technique, it is possible to home GNPs to distinct subcellular compartments using specific peptides such as the nuclear localization sequence and the mitochondrial localization sequence (27). As predicted by the LEM-based modeling described above, greater proximity to the nucleus via nuclear localization of GNPs increases the radiosensitization.

\subsubsection{Radiosensitization}

From a mechanistic standpoint, the effects of irradiating GNPs that are best understood are the increase in DNA damage and oxidative stress. When thin films of plasmid DNA were bombarded with $60 \mathrm{keV}$ electrons in the presence of GNPs in a 1:1 ratio, the formation of DNA single- and double-strand breaks was increased more than two-fold (28). This was attributed to the increased yield of short-range low-energy secondary electrons. Similar results were later confirmed when DNA was in solution and irradiated with orthovoltage x-rays (200 to 500 $k V p)$. Coating $5 \mathrm{~nm}$ GNPs with a $2.5 \mathrm{~nm}$ thick epilayer of thiolated undecane significantly reduced the yield of DNA strand breaks by $30 \%$ compared to uncoated GNPs while other coatings reduced this yield even further, again suggesting that secondary electrons of low energies are principal mediators of DNA damage and are easily attenuated by coatings (29).

This also provides an explanation for size dependence of radiosensitization - the yield of secondary electrons is determined by a trade-off between more gold atoms in the nanoparticle, which increases the Z-dependent photoelectric 
absorption cross-section, and more gold atoms on the surface of the particle attenuating secondary electrons released from ionized atoms in the core of the nanoparticle (self-absorption) (30).

\subsection{In vivo radiosensitization and biodistribution}

In vivo, the determinants of nanoparticle interaction with cell membranes and serum proteins (namely size, shape, charge, and surface functionalization) also dictate the fate of nanoparticles, i.e., their circulation time in the body and the extent of accumulation in the tumor.

\subsubsection{Size and Shape}

Resident macrophages in the reticuloendothelial system (RES) (liver, spleen and lymph nodes) act as filters to eliminate nanoparticles (similar in size to bacteria and viruses) from the body. Nanoparticles of smaller size $(<6 \mathrm{~nm})$ are generally cleared within minutes from systemic circulation via renal excretion after intravenous administration (31). RES accumulation primarily dominates in case of larger nanoparticles, leading to reduced tumor accumulation (32).

A recent study showed that GNPs ranging from 2 to $15 \mathrm{~nm}$ penetrate and localize within cultured cancer cells, multicellular spheroids, and tumors in vivo in a size dependent manner (see figure 4) (33). 2 and 6 nm GNPs demonstrated advantages over larger nanoparticles in terms of tumor uptake in the tumor spheroid model and in vivo, and reduced accumulation in normal tissues.

Circulatory half-lives appear to be a complex product of both particle size and preparation, with particles of a range of sizes often being cleared rapidly from the blood within 24 hours (see Figure 4). A study of $15 \mathrm{~nm}$ and $100 \mathrm{~nm}$ particles showed maximum accumulation in 4-8 hrs followed by gradual decrease over 24 hrs for the larger particles as opposed to continuous increase in accumulation for smaller GNPs (see Figure 7D) (34). Some preparations have reported considerably longer life-times up to 1 week (35) in blood.

The shape of GNPs also influences efficiency of extravasation into tumors - as opposed to spherical particles, elongated particles are more likely to localize along the periphery rather than within the center of an advancing parabolic front of laminar blood flow in a capillary, resulting in a greater probability of contacting the vessel wall and extravasation through vascular fenestrations into the tumor interstitium (36-38). Biodistribution studies on PEGylated gold nanorods and nanospheres showed that the rods saw greater accumulation inside the tumor and had a longer circulation time, consistent with the lesser extent of phagocytosis of these particles by macrophages in vitro (20). Whereas such differences are apparent in individual studies comparing two shapes, these results are specific to the formulation tested, the coating employed, and the model system utilized. They are, therefore, not generalizable to all GNPs of a similar shape.

It was shown that GNPs with a larger amount of PEGs result in a stronger protection of these functional GNPs against clearance through the RES. A 
PEG:gold molar ratio of 1.5 offers an enhanced permeability into tissues and higher retention (39).

Figure 4

Figure 4: Pharmacokinetics and biodistribution of 2, 6, and $15 \mathrm{~nm}$ GNPs coated with tiopronin. A) Blood elimination profiles of gold following a single intravenous injection of GNPs at a dose of $5 \mathrm{mg}$ gold $/ \mathrm{kg}$ in tumor-bearing mice. Data represent mean $\pm S D(n=3)$. B) Gold content in tumor, heart, liver, spleen, lung, brain, and kidney $24 \mathrm{~h}$ after intravenous injection of GNPs at $5 \mathrm{mg}$ gold $/ \mathrm{kg}$. Data represent mean $\pm S D(n=3)$ C) Representative TEM micrographs of tumor tissue taken $24 \mathrm{~h}$ after the administration of GNPs. Figure with permission from (33).

\subsubsection{Surface properties}

The surface functionalization of nanoparticles greatly affects the biodistribution and pharmacokinetics of GNPs in the body. In investigations of PEG-coated GNPs $(35,40,41)$, smaller particles $(5-13 \mathrm{~nm})$ were found to have significant accumulation in the liver. PEG-coated GNPs were also found to have lesser uptake by macrophages (20) or have a potentially longer retention time in circulation (42). In addition, changing the surface charge was observed to determine organ uptake of GNPs (43). The GNP surface charge contributes to electrostatic interactions with serum proteins (in particular the complement factors that opsonize foreign substances and present them to macrophages) and the cell membrane which are both largely negatively charged and greatly influence circulatory half-life of intravenously administered GNPs and their likelihood of internalization in cells.

\section{Targeting tumors}

4.1 Untargeted Gold Nanoparticles

Nanoparticles passively leak into the tumor interstitium from blood vessels feeding the tumor via a phenomenon typically known as "enhanced permeability and retention" (EPR) (44), because of their relatively smaller sizes (typically $<100$ $\mathrm{nm}$ ) compared to the typical cutoff size of the pores (up to $400 \mathrm{~nm}$ ) in the tumor vasculature (45). This property has become the basis of an approach generally known as "passive targeting". Over the last decade, this approach has been adopted by many investigators to improve radiotherapy outcomes with GNPs in small animal studies. Typically, untargeted GNPs were intravenously injected into mice followed by irradiation of tumors using kilovoltage $(2,46)$ or synchrotron (47) X-rays or charged particle beams (electrons (7) and protons (4)), either almost immediately (on the order of minutes) after the GNP injection or at a later time point (on the order of hours to days). X-ray irradiation at the former time 
point results in more damage to the tumor vasculature (i.e. endothelial cells lining the tumor blood vessels) than the tumor cells because, under passive targeting, the tumor gold content is initially an index of vascularity of the tumor, especially following the injection of large amounts of GNPs (e.g., $2.7 \mathrm{~g} \mathrm{gold} / \mathrm{kg}$ )(2). While the majority of GNPs at later time points are expected to have been cleared from the vascular compartment, the remaining extravasated GNPs (via the EPR effect) are typically heterogeneously distributed and found in the perivascular space (48), potentially making disruption of the tumor vasculature still a significant mechanism for the observed radiosensitization effect.

Initial excitement surrounding the use of untargeted GNPs for radiosensitization has been tempered by the recognition that large intratumoral amounts of gold are needed for radiosensitization in vivo. Furthermore, smaller particles (e.g., 1.9nm) (2) act as contrast agents, stay within the vascular compartment, and are rapidly cleared through the kidneys. These hurdles have fueled the quest for alternative formulation and administration strategies.

\subsection{Encapsulated Gold Nanoparticles}

One such strategy invokes the use of liposomes to encapsulate GNPs. Liposomes have been in clinical use for drug encapsulation and delivery for many decades. They are typically $100-200 \mathrm{~nm}$ in size, can be engineered for in vivo stability and long circulation times, and passively accumulate preferentially in tumors via the EPR effect. Their large size ensures that they can pack a large number of GNPs within their lipid bilayer and/or within their cavity. This strategy has been shown to result in substantially higher internalization of $1.4 \mathrm{~nm}$ GNPs within cells than unencapsulated GNPs (49). The mechanism of poorer internalization of the smaller particle than the liposomal particle was postulated to be an unfavorable increase in thermodynamic free energy during membrane wrapping and endocytosis. Extension of this concept in vivo would enable the delivery of greater gold concentrations within tumors than unencapsulated GNPs. 100-200 nm liposomes based formulations are known for passive accumulation by the EPR effect. Because of their long circulation time, small GNPs encapsulated in liposomes showed better passive accumulation than $1.4 \mathrm{~nm}$ GNPs, which are renally excreted within a few minutes. Furthermore, if liposomal contents can be released upon extrinsic activation by a triggering technique (50), this strategy could serve as a Trojan horse that ferries the GNPs within a non-replicating carrier and on-demand deploys its payload of smaller GNPs that can penetrate deeper into tumor parenchyma.

\subsection{Tumor Targeted Gold Nanoparticles}

The motivation for targeting tumor cells preferentially with GNPs is three-fold first, it increases the therapeutic ratio by achieving greater concentrations of nanoparticles within the tumor than surrounding normal tissues; second, it brings the nanoparticle closer to the cellular nucleus for greater DNA damage following radiation therapy; and third it reduces the amount of gold necessary for treatment. "Active targeting" is generally achieved by functionalizing the surface 
of GNPs with peptides or antibodies that dock to cell surface proteins preferentially expressed on cancer cells.

While decoration with a capped PEG molecule enables the GNP to evade capture by the RES, decoration with peptides/antibodies homes the GNP to the tumor. The magnitude of increase in tumor-specific accumulation of activelytargeted nanoparticles compared to untargeted nanoparticles varies depending on the tumor, the targeting moiety, the nanoparticle in question, the relative density of the targeting moiety decorating the surface of the nanoparticle and the time point when the accumulation in the tumor is measured. In general, the magnitude of tumor-specific accumulation achieved with active targeting is less than an order of magnitude greater than with passive targeting.

However, active targeting of GNPs to the tumor cell vs. the stromal fibroblast vs. the vascular endothelium significantly influences the eventual geographical location of the nanoparticle at the tissue level, as well as the internalization and localization of the nanoparticle at the cellular level (51). These two characteristics of active targeting are of particular relevance to using GNPs as radiosensitizers since the location of the nanoparticle within a target cell, as described previously, has a profound influence on the likelihood of inducing a lethal event within it. Early evidence suggests that active targeting achieves these predicted effects with megavoltage photon radiation directed at tumors through tissue-equivalent bolus material. These findings are in agreement with similar findings with conjugated GNPs directly injected intratumorally (52) although achieving satisfactory tumor coverage with direct injection is challenging and may have clinical utility only in limited tumor types. Further enhancement in radiosensitization is potentially achievable with nuclear targeting of GNPs using nuclear localization sequence peptides (27).

Figure 5

Figure 5: Radiosensitization by conjugated GNPs injected intravenously, irradiated with a $1.5 \mathrm{~cm}$ superflab tissue-equivalent bolus covering the irradiated thighs of each mouse. A) TEM images of gold nanorods (AuNRs) conjugated to goserelin, a gonadotropin releasing hormone agonist, demonstrating binding to the cell surface of prostate cancer cells and internalization over time to endocytotic vesicles. B) Clonogenic survival curves demonstrating greater radiosensitization with goserelin-conjugated AuNRs (gAuNRs) than PEGylated AuNRs (pAuNRs) when irradiated with 6MV photons. C) Tumor growth curves of prostate cancer xenografts treated with single dose $6 \mathrm{MV}$ radiation in the presence of AuNRs demonstrating greater delay in tumor regrowth when treated with gAuNRs than pAuNRs. Reprinted with permission from reference 6 .

\subsection{Vascular Targeted Gold Nanoparticles}


The tumor vasculature has been identified as a potentially important target in cancer therapy. Both anti-angiogenic and vascular disruptive approaches have been explored to control tumor growth and starve tumor cells. While chemical vascular disruptive agents (VDA) have been shown to be efficacious in human clinical trials, excessive toxicities due to systemic administration have been a barrier to widespread usage (53-56).

However, it has been shown that targeting tumor blood vessels with drugs or radiopharmaceuticals has high therapeutic value. As examples, Murphy et al. have shown that nanoparticle-mediated drug delivery to tumor vasculature can even have an anti-metastatic effects (57) and pre-clinical studies showed that radio-pharmaceuticals concentrated around blood vessels are more effective than a uniform distribution within the tumor (58). Similarly, for radiation therapy combined with GNPs, a higher concentration of GNPs near the vasculature could provide a biological advantage over a homogeneous distribution throughout the tumor.

The synergy between chemical VDAs and radiation therapy has been demonstrated (59-61). Accumulation of GNPs in the vasculature is expected for nanoparticles of a certain size (sub-100nm diameter (62)) and can be further improved by molecular targeting to tumor blood vessels. Combining the tumor targeting with precise image-guided radiation therapy allows the GNP-enriched tumor to be targeted. A localized vascular-disrupting therapy can be delivered much more safely in combination with radiation therapy than is currently achievable with VDA.

Most chemotherapy approaches rely on diffusion of the agent into the tumor. While the EPR effect does improve the reach of these agents for tumors with leaky blood vessels, relying on passive diffusion to provide a homogenous distribution is challenging. Conversely, poor diffusion is a boon for tumor vascular targeted therapies. In fact, nanoparticles can be designed to limit diffusion and, linked with vascular targeting agents, to further ensure their close proximity to blood vessel endothelial cells. Joh et al. demonstrated "vascular dose painting" with GNPs and $175 \mathrm{kVp}$ photons in a preclinical GBM model (63). Theoretical calculations, combining Monte Carlo with an analytical microdosimetry calculations $(64,65)$ predict a roughly $50 \%-150 \%$ increase in dose to the tumor endothelial cells by a standard $6 \mathrm{MV}$ beam, and softening of MV photon spectrum, as mentioned earlier, further increases such dose enhancement.

While the concept of using GNPs as VDA is enticing, there are still several issues left to be resolved. Clinical timing of injections relative to radiation delivery, sufficient accumulation in tumor blood vessels, and the secondary or tertiary effects of tumor blood vessel disruption (e.g. increased hypoxia) need to be investigated.

\subsection{GNP fabrication}

The foregoing discussion outlines the need for rigorous characterization of nanoparticles in vitro and in vivo prior to embarking on clinical trials. Failure to optimize these parameters during fabrication of GNP-based therapeutic platforms may results in undesirable side effects when GNPs are used in conjunction with 
radiation i.e. potential radiosensitization of the non-targeted RES organs. Engineering a successful nanoparticle based platform must take into account the type and microenvironment of the disease, the fate of these materials in the in vivo environment and how these nanoparticles will interact with non-target organs. Thus, learning from the drawbacks associated with previous generations of nanoparticles, next generation nanoparticles may be engineered to improve the therapeutic index with minimized toxicity.

\section{Clearance and Toxicity}

The blood circulation times of nanoparticles were found dependent on nanoparticle (NP) size and the chain length of the protective PEG coat $(42,66)$. For example, a previous study by Cho et al observed the biodistribution of PEGcoated GNPs in an in vivo mice model, all of which were healthy (66). It was found that within 7 days of intravenous application, 4 and $13 \mathrm{~nm}$ NPs were distributed to the RES organs such as the liver and spleen. The concentration of these small NPs in the RES organs peaked at 7 days followed by decreasing gold amounts. On the other hand, $100 \mathrm{~nm}$ NPs left the blood within 30 minutes of intravenous application followed by distribution to the RES organs. However, the concentration in the RES organs peaks at 30 minutes but remains high for 6 months. Hence, assuming the amount of PEG molecules were the same for all sizes of NPs, small NPs will have longer blood circulation times and will be cleared from the RES organs faster, while large NPs will have shorter blood circulation times and will be cleared from the RES organs slower.

A previous study by Lipka et al also observed the biodistribution of PEG-coated gold NPs in an in vivo mice model, which were healthy, except the core size of NPs was constant at $5 \mathrm{~nm}$ in diameter. Various coatings were applied, which included phosphate, short PEG chains (750 Da) and long PEG chains $(10,000$ $\mathrm{Da})$. It was observed that within an hour of intravenous application, less than $5 \%$ of phosphate-coated and short PEG chain-coated NPs were found in the blood, while the rest were distributed in the RES organs. On the other hand, over $90 \%$ of NPs coated with long PEG chains remained in the blood and avoided being distributed to RES organs for clearance. Hence, longer PEG chain lengths promote long blood circulation times. The biocompatibility of the coating ligands on the nanoparticles' surface is a key consideration for all biomedical applications(40).

Similar to the discussion about determinants of uptake and radiosensitization, toxicity of GNPs is also a function of concentration, size, shape, surface charge and surface functionalization. High concentrations of $4 \mathrm{~nm}$ GNPs reduce cell viability mostly through induction of reactive oxygen species, noted at concentrations of $50 \mathrm{nM}$ or higher (see figure 6) (67). At $10 \mathrm{nM}$ concentration of GNPs, no significant effects on cell morphology, cytoskeleton architecture, or cell functionality were observed. These data highlight the importance of using multiple assays to cover the broad spectrum of GNP interactions with cells and to determine safe GNP concentrations. 
Figure 6

Figure 6: A) Representative confocal images of control HUVECs or cells exposed to 10, 20, 50, or $100 \mathrm{nM}$ GNP for $24 \mathrm{~h}$ at 1 day post-NP-labeling. The left column depicts actin staining (red), the middle column depicts $\alpha$-tubulin (green), and the right column is a merged image of both actin and $\alpha$-tubulin. Scale bars: $50 \mu \mathrm{m}$. B) Histograms representing the cell areas of control cells (dark gray) and cells incubated for $24 \mathrm{~h}$ with GNPs at $10 \mathrm{nM}$ (B1), $20 \mathrm{nM}$ (B2), $50 \mathrm{nM}$ (B3), and 100 $n M(B 4)$. The average cell area is indicated with (*) for control cells and with (§) for NP-treated cells. Figure with permission from (67).

Other studies have shown GNPs to be cytotoxic due to their inherent physicochemical properties. In investigations of PEG-coated GNPs $(35,40,41)$, smaller particles $(5-13 \mathrm{~nm})$ were found to have significant accumulation in the liver, with $13 \mathrm{~nm}$ particles causing acute inflammation and apoptosis in the liver. These GNPs were preferentially taken up by Kupffer cells Kupffer cells and hepatocytes. They also transiently activated phase I metabolic enzymes in liver tissues from $24 \mathrm{~h}$ to 7 days, whereas $100 \mathrm{~nm}$ GNPs did not. However, Huang et al (33) have used GNPs of size 2, 6, and $15 \mathrm{~nm}$, but did not report such toxicity issues. These GNPs were coated with tiopronin. Hence, the toxicity could not only be dependent of the size but also due to the surface properties.

The GNP size plays a significant role in various physiological processes (hepatic filtration, renal excretion, tissue extravasation and diffusion). It has been well documented that the decrease in the size of the nanoparticle correlates with the increase in the nanoparticle's capacity to navigate between the tumor interstitium after extravasation. The nanoparticles should have a design that shows a maximal tumor uptake with minimal uptake in the RES yet have long circulating properties and should be cleared via the renal excretion upon disintegration of the surface ligands. The previously reported GNP formulation in which the hydrodynamic size of the PEGylated GNPs formulation was $12 \mathrm{~nm}$ (core size $\sim 2 \mathrm{~nm}$ ) presents one such example of a good GNP-based system as the hydrodynamic diameter will avoid the faster renal excretion with lower hepatic infiltration (68). Further, the degradation of the PEG coating from the GNP surface may lead to renal clearance, however, the human body does not have enzymes to effectively degrade larger PEGs. The GNP-based formulations with similar size and surface properties, where the pharmacokinetics can be modulated by tuning the physico-chemical properties, have high potential in providing greater therapeutic benefits with reduced toxicities.

The surface charge of GNPs has also been shown to be important in determining particle toxicity, with cationic GNPs exhibiting moderate toxicity owing to the electrostatic binding of the particles to the negatively charged cell membrane. In contrast, anionic particles have less toxicity as they are repelled from the membrane (69). 


\section{Visualizing in vitro and in vivo uptake of GNPs}

Different biomedical imaging modalities are capable of providing not only anatomical imaging (e.g., whole body MRI) but also sub-cellular level resolution (e.g., optical imaging). GNPs' inherent properties can be exploited for visualization by conventional CT (70) and X-ray Fluorescence Computed Tomography (XFCT) (71), photo-acoustic imaging/tomography (72), and Surface Enhanced Raman Scattering (SERS)(70, 73). Some of these modalities can be performed in the same platform by taking advantages of complementary nature in the imaging modality and geometry (e.g., conventional CT and XFCT/optical imaging) and the ease of fabrication of GNP-based formulations (e.g., optical and MR imaging). The versatility of next generation GNP platforms allows for conjugation to organic fluorophores as well as chelating agents like gadolinium to impart dual imaging capabilities to GNPs for both in vitro and in vivo labeling and quantification. As noted above, once GNPs are optimally fabricated, functionalized and administered intravenously, the potential for and the magnitude of radiosensitization depends on the concentration of gold within the tumor compared to adjacent normal tissues and the geographical distribution of the GNPs within the tumor.

In order to properly monitor the intratumoral distribution of GNPs during preclinical studies on GNP-mediated radiosensitization, imaging modalities capable of detecting trace amount of GNPs on the order of parts-per-million (ppm)(e.g., $10 \mu \mathrm{g}$ gold $/ \mathrm{g})$ would be necessary. Optical imaging (74-76), photo-acoustic imaging $(72,77)$ and benchtop XFCT $(78)$ have been demonstrated to meet this requirement. Conventional (or transmission) CT as implemented in micro-CTs can be used for the same purpose $(79,80)$, but the system sensitivity is typically on the order of $1 \mathrm{mg} \mathrm{gold} / \mathrm{g}$. MRI or single-photon emission CT (SPECT) can also be used to detect GNPs conjugated with MR-visible agents (e.g., gadolinium) $(81,82)$ or gamma-emitting nuclides (e.g., In-111) (83),, respectively. In general, SPECT is considered capable of detecting ppm-level of radiolabeled GNPs in vivo, whereas MRI may need to be performed with a high field strength (e.g., 7T) scanner to detect MR-visible GNPs at such a low concentration.

\subsection{In vitro imaging}

In vitro imaging using GNPs can illustrate the mechanism of cellular uptake by endocytosis, and also determine whether the nanoparticles are specifically targeted to the nucleus (to inflict the maximum DNA damage) or for uptake in cytoplasm. These observations will provide important insights in developing better GNP formulations. The conjugation of fluorophores imparts optical imaging capabilities to GNPs as noted earlier (68). Due to enhancement of the optical field at the surface of nanoparticles, GNPs can be observed using multi-photon luminescence, this phenomenon has been used to observe GNP uptake in embryonic stem cells (84). Due to their high electron density, GNPs can easily be visualized under Transmission/Scanning Electron Microcopy (TEM/SEM), enabling easy localization within fixed sections (85). 


\subsection{In vivo imaging}

Due to the high attenuation of X-rays by gold, GNPs have already been studied as efficient multimodal imaging contrast agents (34) (81). Conjugating gadolinium provides additional contrast capability for MRI (82). A dual mode imaging platform combining optical and CT imaging using GNPs has been reported earlier for in vivo applications (86). While MRI provides superior in vivo anatomical information about the accumulation of GNPs in the tumor, optical and electron microscopies are necessary to study the sub-cellular distribution of GNPs in the tumor. Collectively, both in vitro and in vivo imaging are important tools to optimize GNPs for radiosensitization applications.

\subsection{Quantitative in vivo imaging with benchtop XFCT}

Besides the aforementioned in vivo imaging modalities, benchtop XFCT has emerged in recent years as a promising quantitative imaging modality that is, in principle, capable of simultaneously determining the amount and spatial distribution of GNPs in vivo. The basic proof-of-principle of benchtop XFCT has already been established (see figure 7$)(71,78,87,88)$. While it has yet to be demonstrated through in vivo studies, benchtop XFCT may enable direct and non-invasive visualization of GNP distributions in vivo, resulting in more accurate determination and monitoring of the biodistribution and targeted delivery of GNPs.

\section{Figure 7}

Figure 7: A) Photoacoustic images of tumors in vivo before (top) and $6 \mathrm{~h}$ after (bottom) intravenous administration of gold nanorods documenting extravasation of nanoparticles from vasculature to tumor parenchyma. Reproduced with permission from reference (77). B) XFCT imaging phantom with simulated GNPladen blood vessel and $\sim 1 \mathrm{~cm}^{3}$ tumor. The box shows the region of interest that was imaged. C) 2-D $x$-ray fluorescence image of the region of interest within the $X F C T$ imaging phantom. The GNP distribution within the simulated tumor region and blood vessels is shown, with the maximum value of $\sim 5.5 \mu \mathrm{g}$. The outline of the irradiated GNP-containing structures is overlaid on the fluorescence image, showing a good match between the images around the tumor region. From reference (78) with permission (pending). D) Fluorescence image guided biodistribution studies with GNPs of MDA-MB-435 tumors (yellow arrows) in mice injected simultaneously with $15 \mathrm{~nm}$ (blue fluorescence) and $100 \mathrm{~nm}$ (red fluorescence) GNPs which were coated with PEG $5 \mathrm{kDa}$, and fluorescently labelled with Kodak X-Sight 670 and Alexa Fluor 750, respectively, from reference (34) with permission (pending). 


\section{How can GNPs become part of clinical routine?}

The versatility of design and function of nanoparticles can be exploited in a number of ways to augment radiation therapy via radiation dose enhancement as outlined above. Preclinical studies have been largely confined to proof-ofprinciple experiments and modeling. Nevertheless, there is a convergence of results across multiple studies that set the stage for realizing tangible therapeutic gains in clinical radiotherapy.

As outlined in the NCl-RTOG translational program strategic guidelines for the early-stage development of radiosensitizers, the path to clinical translation of any radiosensitizer is never a seamless transition from the bench to the bedside (89). However, some guiding principles provide a framework for clinical translation. Appropriate patient selection is a pivotal first step in clinical translation. The ideal scenario where potential gains from GNP-mediated radiosensitization can be evaluated would be tumors that are treated definitively or pre-operatively with radiation therapy or chemoradiation therapy, benefit from dose escalation focally to the tumor but where such dose escalation is not feasible by conventional means due to normal tissue constraints, express specific cell surface receptors that can be targeted with peptides or antibodies, and where current standard-ofcare treatment yields unsatisfactory results. This would include tumors of the brain, prostate, lung, pancreas, as well as many head \& neck sites. Within a given tumor type, the selection of the optimal clinical context to test a GNPmediated radiosensitization strategy would also need to be judiciously considered. For instance, since clinical standards of care for many tumors treated with radiation include the concurrent administration of chemotherapy, integration of GNP radiosensitization into this workflow will require demonstration of either (i) equivalent efficacy and a favorable toxicity profile of GNP + radiation compared to chemoradiation or (ii) an additive or synergistic benefit when GNPs are combined with standard chemoradiation. Preclinical data in this realm is currently sparse. Early studies of DNA damage in solution suggest that GNPs have the ability to potently sensitize chemoradiation therapy (90). More rigorous preclinical studies evaluating combination therapy with chemotherapeutic agents and radiation therapy would be necessary before embarking on clinical studies. On the other hand, even in tumors where concurrent chemotherapy is not the standard of care, development of radiosensitization strategies could benefit from enrichment of the study population with patients who are most likely to benefit from GNP-mediated radiosensitization. At this time, there are no biomarkers of response to GNP-mediated radiosensitization to guide a biomarker-guided selection process but clinical predictors of high risk of local recurrence could serve as a means of study population enrichment.

Aside from appropriate patient selection, a key consideration is the choice of the specific GNP and its preclinical validation. Meticulous characterization of physicochemical properties, biodistribution and pharmacokinetics, and molecular mechanisms would be mandatory prerequisites for seamless and smooth translation from bench to bedside. The GNP design should reflect practical 
clinical considerations. GNPs should provide a long biological half-life, enabling them to be delivered on a more flexible schedule than immediately before each fraction. This would require a combination of GNP size (likely in the range 2 to 50 $\mathrm{nm}$ ), coating (likely PEG) and a (most likely tumor-specific) targeting moeity (likely peptide or anti-body based) that offers good tumor homing and long biological half-life. Unlike classical pharmaceutical sensitizers of radiation therapy, nanoparticle-mediated radiosensitization offers the possibility and the promise of being able to directly quantify and visualize their accumulation within tumors thereby aiding image-guided therapy and quantitative dosimetry where physical and biological consequences could be modeled and predicted before treatment. Like pharmaceutical radiosensitizers, combination with other agents would need to be evaluated prior to embarking on clinical studies - early studies of DNA damage in solution suggest that GNPs have the ability to potently sensitize chemoradiation therapy (90). As with all investigational new drugs, GNP formulations being proposed for clinical evaluation will need to undergo rigorous testing to ensure their immediate and long-term safety and tolerability in humans, despite the general notion that gold is relatively non-toxic and biocompatible. This evaluation could be undertaken jointly with the Nanotechnology Characterization Laboratory at $\mathrm{NCl}$ which serves as the national resource base facilitating regulatory review of nanotechnologies intended for cancer therapy. Lastly, since safety and affordability are dictated by the amount of GNPs infused into the human body, the formulations should achieve desired radiation dose enhancement with small quantities of gold.

For optimum clinical translation, GNPs should be introduced where they address a current clinical need without a significant change in the clinical approach. The greatest clinical benefit of GNPs may be achieved with a treatment system that is optimized for GNP radiosensitization using keV photons. However, a timely transition of GNPs into clinical practice will likely utilize radiation types that offer sensitization in therapies, which are commonly used clinically - megavoltage radiation, charged particles or brachytherapy. The need to validate a novel treatment radiotherapy technique alongside the GNPs would significantly complicate their introduction and clinical evaluation.

We think many of the prerequisites outlined above for an ideal nanoparticle design are likely to be met by multiple formulations and only with further studies will we be able to decide which formulation or combination of formulations to advance to clinical testing and in what clinical scenario to perform the pilot clinical trial(s). Only after such clinical evaluation would it be practical to consider advancing newer strategies that incorporate drug and/or oligonucleotide delivery using the same platform.

\section{Summary}

Despite the existence of nanoparticle formulations for centuries, it is only in the last decade that we have seen significant advancement and refinement of strategies to employ GNPs as radiosensitizers. The recent profusion of interest and maturity of scientific inquiry provides us a unique opportunity to collectively advance translation of novel preclinical findings from the bench to the bedside. 
We envision that some, if not many, of these research efforts will develop further in the near future to impact clinical care. A distinctive feature of such efforts will be the interdisciplinary nature of the collaborations required since this paradigm of radiation dose enhancement using GNPs sits at the intersection of as disparate scientific disciplines as clinical radiation oncology, radiation physics and biology, nanotechnology, materials science, biomedical engineering, radiographic imaging, pharmacology, chemistry, and tumor biology. We believe that tapping the collective wisdom of practitioners of these disciplines in academia, industry and federal regulatory bodies will expedite the rational progression from robust preclinical data to first in-human clinical trials that have the potential to revolutionize radiation therapy.

Tables 1-5 summarize the pathway to a possible clinical implementation of GNP enhanced radiation therapy.

Table 1: Pathway to implementing GNPS as clinical agents.

Table 1

Table 2: Characteristics of tumor sites suitable for GNP-enhanced radiation therapy

Table 2

Table 3: Important characteristics of GNPs for treatment enhancement

Table 3

Table 4: Pre-clinical experimental results needed to inform development of GNPbased therapies 
Table 4

Table 5: Clinical experimental results required for final validation of GNPenhanced radiotherapy

Table 5

\section{References:}

1. Santos Mello R, Callisen $\mathrm{H}$, Winter $\mathrm{J}$, et al. Radiation dose enhancement in tumors with iodine. Med. Phys. 1983;10:75-78.

2. Hainfeld JF, Slatkin DN, Smilowitz HM. The use of gold nanoparticles to enhance radiotherapy in mice. Phys. Med. Biol. 2004;49:N309-15.

3. Butterworth KT, McMahon SJ, Currell FJ, et al. Physical basis and biological mechanisms of gold nanoparticle radiosensitization. Nanoscale. 2012;4:4830.

4. Kim J-K, Seo S-J, Kim H-T, et al. Enhanced proton treatment in mouse tumors through proton irradiated nanoradiator effects on metallic nanoparticles. Phys. Med. Biol. 2012;57:8309-8323.

5. Liu C-J, Wang C-H, Chien C-C, et al. Enhanced x-ray irradiation-induced cancer cell damage by gold nanoparticles treated by a new synthesis method of polyethylene glycol modification. Nanotechnology. 2008;19:295104. 
6. Wolfe T, Chatterjee D, Lee J, et al. Targeted gold nanoparticles enhance sensitization of prostate tumors to megavoltage radiation therapy in vivo. Nanomedicine. 2015.

7. Zhang X, Xing JZ, Chen J, et al. Enhanced radiation sensitivity in prostate cancer by gold-nanoparticles. Clin Invest Med. 2008;31:E160-7.

8. Cho SH. Estimation of tumour dose enhancement due to gold nanoparticles during typical radiation treatments: a preliminary Monte Carlo study. Phys. Med. Biol. 2005;50:N163-73.

9. Cho SH, Jones BL, Krishnan S. The dosimetric feasibility of gold nanoparticleaided radiation therapy (GNRT) via brachytherapy using low-energy gamma-/xray sources. Phys. Med. Biol. 2009;54:4889-4905.

10. McMahon SJ, Hyland WB, Muir MF, et al. Biological consequences of nanoscale energy deposition near irradiated heavy atom nanoparticles. Sci Rep. $2011 ; 1: 18$.

11. Lin Y, McMahon SJ, Scarpelli M, et al. Comparing gold nano-particle enhanced radiotherapy with protons, megavoltage photons and kilovoltage photons: a Monte Carlo simulation. Phys. Med. Biol. 2014;59:7675-7689.

12. Jones BL, Krishnan S, Cho SH. Estimation of microscopic dose enhancement factor around gold nanoparticles by Monte Carlo calculations. Med. Phys. 2010;37:3809.

13. Montenegro M, Nahar SN, Pradhan AK, et al. Monte Carlo simulations and atomic calculations for Auger processes in biomedical nanotheranostics. $J$ Phys Chem A. 2009;113:12364-12369.

14. Robar JL, Riccio SA, Martin MA. Tumour dose enhancement using modified megavoltage photon beams and contrast media. Phys. Med. Biol. 2002;47:24332449.

15. Tsiamas $\mathrm{P}$, Liu B, Cifter $\mathrm{F}$, et al. Impact of beam quality on megavoltage radiotherapy treatment techniques utilizing gold nanoparticles for dose enhancement. Phys. Med. Biol. 2013;58:451-464.

16. Perl J, Shin J, Schuemann J, et al. TOPAS: An innovative proton Monte Carlo platform for research and clinical applications. Med. Phys. 2012;39:6818.

17. McMahon SJ, Butterworth KT, McGarry CK, et al. A computational model of cellular response to modulated radiation fields. Int. J. Radiat. Oncol. Biol. Phys. 2012;84:250-256.

18. Lin $\mathrm{Y}$, McMahon SJ, Paganetti $\mathrm{H}$, et al. Biological modeling of gold nanoparticle enhanced radiotherapy for proton therapy. Phys. Med. Biol. 
2015;60:4149-4168.

19. Butterworth KT, Coulter JA, Jain S, et al. Evaluation of cytotoxicity and radiation enhancement using $1.9 \mathrm{~nm}$ gold particles: potential application for cancer therapy. Nanotechnology. 2010;21:295101.

20. Arnida, Janát-Amsbury MM, Ray A, et al. Geometry and surface characteristics of gold nanoparticles influence their biodistribution and uptake by macrophages. Eur J Pharm Biopharm. 2011;77:417-423.

21. Chithrani BD, Ghazani AA, Chan WCW. Determining the size and shape dependence of gold nanoparticle uptake into mammalian cells. Nano Lett. 2006;6:662-668.

22. Chithrani DB, Jelveh S, Jalali F, et al. Gold Nanoparticles as Radiation Sensitizers in Cancer Therapy. Radiation Research. 2010;173:719-728.

23. Gao H, Shi W, Freund LB. Mechanics of receptor-mediated endocytosis. Proc. Natl. Acad. Sci. U.S.A. 2005;102:9469-9474.

24. Carter JD, Cheng NN, Qu Y, et al. Nanoscale energy deposition by X-ray absorbing nanostructures. J Phys Chem B. 2007;111:11622-11625.

25. Champion JA, Katare YK, Mitragotri S. Particle shape: a new design parameter for micro- and nanoscale drug delivery carriers. J Control Release. 2007;121:3-9.

26. Kah JCY, Wong KY, Neoh KG, et al. Critical parameters in the pegylation of gold nanoshells for biomedical applications: an in vitro macrophage study. J Drug Target. 2009;17:181-193.

27. Yang C, Uertz J, Yohan D, et al. Peptide modified gold nanoparticles for improved cellular uptake, nuclear transport, and intracellular retention. Nanoscale. 2014;6:12026-12033.

28. Zheng Y, Hunting DJ, Ayotte P, et al. Radiosensitization of DNA by gold nanoparticles irradiated with high-energy electrons. Radiation Research. 2008;169:19-27.

29. Xiao F, Zheng Y, Cloutier $P$, et al. On the role of low-energy electrons in the radiosensitization of DNA by gold nanoparticles. Nanotechnology. $2011 ; 22: 465101$.

30. Leung MKK, Chow JCL, Chithrani BD, et al. Irradiation of gold nanoparticles by $\mathrm{x}$-rays: Monte Carlo simulation of dose enhancements and the spatial properties of the secondary electrons production. Med. Phys. 2011;38:624-631.

31. Choi HS, Liu W, Misra P, et al. Renal clearance of quantum dots. Nature 
Biotechnology. 2007;25:1165-1170.

32. Kumar R, Roy I, Ohulchanskky TY, et al. In vivo biodistribution and clearance studies using multimodal organically modified silica nanoparticles. ACS Nano. 2010;4:699-708.

33. Huang $\mathrm{K}, \mathrm{Ma} \mathrm{H}$, Liu J, et al. Size-dependent localization and penetration of ultrasmall gold nanoparticles in cancer cells, multicellular spheroids, and tumors in vivo. ACS Nano. 2012;6:4483-4493.

34. Chou LYT, Chan WCW. Fluorescence-tagged gold nanoparticles for rapidly characterizing the size-dependent biodistribution in tumor models. Adv Healthc Mater. 2012;1:714-721.

35. Cho W-S, Cho M, Jeong J, et al. Acute toxicity and pharmacokinetics of 13 nm-sized PEG-coated gold nanoparticles. Toxicol. Appl. Pharmacol. 2009;236:16-24.

36. Geng Y, Dalhaimer P, Cai S, et al. Shape effects of filaments versus spherical particles in flow and drug delivery. Nat Nanotechnol. 2007;2:249-255.

37. Pluen A, Netti PA, Jain RK, et al. Diffusion of macromolecules in agarose gels: comparison of linear and globular configurations. Biophysical journal. 1999;77:542-552.

38. Lee S-Y, Ferrari M, Decuzzi P. Shaping nano-/micro-particles for enhanced vascular interaction in laminar flows. Nanotechnology. 2009;20:495101.

39. Tiwari P, Vig K, Dennis V, et al. Functionalized Gold Nanoparticles and Their Biomedical Applications. Nanomaterials 2011, Vol. 1, Pages 31-63. 2011;1:3163.

40. Verma A, Uzun O, Hu Y, et al. Surface-structure-regulated cell-membrane penetration by monolayer-protected nanoparticles. Nat Mater. 2008;7:588-595.

41. Zhang X-D, Wu D, Shen X, et al. Size-dependent radiosensitization of PEGcoated gold nanoparticles for cancer radiation therapy. Biomaterials. 2012;33:6408-6419.

42. Lipka J, Semmler-Behnke M, Sperling RA, et al. Biodistribution of PEGmodified gold nanoparticles following intratracheal instillation and intravenous injection. Biomaterials. 2010;31:6574-6581.

43. Hirn S, Semmler-Behnke M, Schleh C, et al. Particle size-dependent and surface charge-dependent biodistribution of gold nanoparticles after intravenous administration. European Journal of Pharmaceutics and Biopharmaceutics. 2011;77:407-416. 
44. Maeda $\mathrm{H}$, Fang J, Inutsuka $\mathrm{T}$, et al. Vascular permeability enhancement in solid tumor: various factors, mechanisms involved and its implications. Int. Immunopharmacol. 2003;3:319-328.

45. Unezaki S, Maruyama K, Hosoda J-I, et al. Direct measurement of the extravasation of polyethyleneglycol-coated liposomes into solid tumor tissue by in vivo fluorescence microscopy. International Journal of Pharmaceutics. 1996;144:11-17.

46. Hainfeld JF, Smilowitz HM, O'Connor MJ, et al. Gold nanoparticle imaging and radiotherapy of brain tumors in mice. Nanomedicine (Lond). 2013;8:16011609.

47. Hainfeld JF, Dilmanian FA, Zhong Z, et al. Gold nanoparticles enhance the radiation therapy of a murine squamous cell carcinoma. Phys. Med. Biol. 2010;55:3045-3059.

48. Diagaradjane P, Shetty A, Wang JC, et al. Modulation of in vivo tumor radiation response via gold nanoshell-mediated vascular-focused hyperthermia: characterizing an integrated antihypoxic and localized vascular disrupting targeting strategy. 2008;8:1492-1500.

49. Chithrani DB, Dunne M, Stewart J, et al. Cellular uptake and transport of gold nanoparticles incorporated in a liposomal carrier. Nanomedicine. 2010;6:161169.

50. Kneidl B, Peller M, Winter G, et al. Thermosensitive liposomal drug delivery systems: state of the art review. Int J Nanomedicine. 2014;9:4387-4398.

51. Huang $X$, Peng $X$, Wang $Y$, et al. A reexamination of active and passive tumor targeting by using rod-shaped gold nanocrystals and covalently conjugated peptide ligands. ACS Nano. 2010;4:5887-5896.

52. Chattopadhyay N, Cai Z, Kwon YL, et al. Molecularly targeted gold nanoparticles enhance the radiation response of breast cancer cells and tumor xenografts to X-radiation. Breast Cancer Res. Treat. 2013;137:81-91.

53. Park HJ, Griffin RJ, Hui S, et al. Radiation-induced vascular damage in tumors: implications of vascular damage in ablative hypofractionated radiotherapy (SBRT and SRS). Radiation Research. 2012;177:311-327.

54. Siemann DW. The unique characteristics of tumor vasculature and preclinical evidence for its selective disruption by Tumor-Vascular Disrupting Agents. Cancer Treat. Rev. 2011;37:63-74.

55. Siemann DW, Horsman MR. Vascular targeted therapies in oncology. Cell Tissue Res. 2009;335:241-248. 
56. Siemann DW, Mercer E, Lepler S, et al. Vascular targeting agents enhance chemotherapeutic agent activities in solid tumor therapy. Int. J. Cancer. 2002;99:1-6.

57. Murphy EA, Majeti BK, Barnes LA, et al. Nanoparticle-mediated drug delivery to tumor vasculature suppresses metastasis. Proc. Natl. Acad. Sci. U.S.A. 2008;105:9343-9348.

58. Boerman OC, Sharkey RM, Blumenthal RD, et al. The presence of a concomitant bulky tumor can decrease the uptake and therapeutic efficacy of radiolabeled antibodies in small tumors. Int. J. Cancer. 1992;51:470-475.

59. Murata R, Siemann DW, Overgaard J, et al. Improved tumor response by combining radiation and the vascular-damaging drug 5,6-dimethylxanthenone-4acetic acid. Radiation Research. 2001;156:503-509.

60. Siemann DW, Rojiani AM. The vascular disrupting agent ZD6126 shows increased antitumor efficacy and enhanced radiation response in large, advanced tumors. Radiation Oncology Biology. 2005;62:846-853.

61. Wilson WR, Li AE, Cowan DS, et al. Enhancement of tumor radiation response by the antivascular agent 5,6-dimethylxanthenone-4-acetic acid. Radiation Oncology Biology. 1998;42:905-908.

62. Perrault SD, Walkey $\mathrm{C}$, Jennings $\mathrm{T}$, et al. Mediating tumor targeting efficiency of nanoparticles through design. Nano Lett. 2009;9:1909-1915.

63. Joh DY, Sun L, Stangl M, et al. Selective targeting of brain tumors with gold nanoparticle-induced radiosensitization. Castro MG, ed. PLOS ONE. 2013;8:e62425.

64. Berbeco RI, Ngwa W, Makrigiorgos GM. Localized dose enhancement to tumor blood vessel endothelial cells via megavoltage X-rays and targeted gold nanoparticles: new potential for external beam radiotherapy. Int. J. Radiat. Oncol. Biol. Phys. 2011;81:270-276.

65. Detappe A, Tsiamas P, Ngwa W, et al. The effect of flattening filter free delivery on endothelial dose enhancement with gold nanoparticles. Med. Phys. 2013;40:031706.

66. Cho W-S, Cho M, Jeong J, et al. Size-dependent tissue kinetics of PEGcoated gold nanoparticles. Toxicol. Appl. Pharmacol. 2010;245:116-123.

67. Soenen SJ, Manshian B, Montenegro JM, et al. Cytotoxic effects of gold nanoparticles: a multiparametric study. ACS Nano. 2012;6:5767-5783.

68. Kumar R, Korideck H, Ngwa W, et al. Third generation gold nanoplatform optimized for radiation therapy. Transl Cancer Res. 2013;2. 
69. Goodman CM, McCusker CD, Yilmaz T, et al. Toxicity of gold nanoparticles functionalized with cationic and anionic side chains. Bioconjug. Chem. 2004;15:897-900.

70. Kim D, Park S, Lee JH, et al. Antibiofouling polymer-coated gold nanoparticles as a contrast agent for in vivo $\mathrm{X}$-ray computed tomography imaging. J. Am. Chem. Soc. 2007;129:7661-7665.

71. Cheong S-K, Jones BL, Siddiqi AK, et al. X-ray fluorescence computed tomography (XFCT) imaging of gold nanoparticle-loaded objects using $110 \mathrm{kVp}$ x-rays. Phys. Med. Biol. 2010;55:647-662.

72. Agarwal A, Huang SW, O'Donnell M, et al. Targeted gold nanorod contrast agent for prostate cancer detection by photoacoustic imaging. Journal of Applied Physics. 2007;102:064701.

73. Qian X-M, Nie SM. Single-molecule and single-nanoparticle SERS: from fundamental mechanisms to biomedical applications. Chem Soc Rev. 2008;37:912-920.

74. Huang X, El-Sayed MA. Gold nanoparticles: Optical properties and implementations in cancer diagnosis and photothermal therapy. Journal of Advanced Research. 2010;1:13-28.

75. Sokolov K, Follen M, Aaron J, et al. Real-time vital optical imaging of precancer using anti-epidermal growth factor receptor antibodies conjugated to gold nanoparticles. Cancer Research. 2003;63:1999-2004.

76. la Zerda de A, Prabhulkar S, Perez VL, et al. Optical coherence contrast imaging using gold nanorods in living mice eyes. Clin. Experiment. Ophthalmol. 2014:n/a-n/a.

77. Jokerst JV, Cole AJ, Van de Sompel D, et al. Gold nanorods for ovarian cancer detection with photoacoustic imaging and resection guidance via Raman imaging in living mice. ACS Nano. 2012;6:10366-10377.

78. Manohar N, Reynoso FJ, Cho SH. Experimental demonstration of direct Lshell $x$-ray fluorescence imaging of gold nanoparticles using a benchtop x-ray source. Med. Phys. 2013;40:080702.

79. Hainfeld JF, O'Connor MJ, Dilmanian FA, et al. Micro-CT enables microlocalisation and quantification of Her2-targeted gold nanoparticles within tumour regions. Br J Radiol. 2011;84:526-533.

80. Clark DP, Ghaghada K, Moding EJ, et al. In vivo characterization of tumor vasculature using iodine and gold nanoparticles and dual energy micro-CT. Phys. Med. Biol. 2013;58:1683-1704. 
81. Park J-A, Reddy PAN, Kim H-K, et al. Gold nanoparticles functionalised by Gd-complex of DTPA-bis(amide) conjugate of glutathione as an MRI contrast agent. Bioorg. Med. Chem. Lett. 2008;18:6135-6137.

82. Miladi I, Alric $\mathrm{C}$, Dufort $\mathrm{S}$, et al. The in vivo radiosensitizing effect of gold nanoparticles based MRI contrast agents. Small. 2014;10:1116-1124.

83. Zhang G, Yang Z, Lu W, et al. Influence of anchoring ligands and particle size on the colloidal stability and in vivo biodistribution of polyethylene glycolcoated gold nanoparticles in tumor-xenografted mice. Biomaterials. 2009;30:1928-1936.

84. Nagesha D, Laevsky GS, Lampton $P$, et al. In vitro imaging of embryonic stem cells using multiphoton luminescence of gold nanoparticles. Int $J$ Nanomedicine. 2007;2:813-819.

85. Chithrani DB. Intracellular uptake, transport, and processing of gold nanostructures. Mol. Membr. Biol. 2010;27:299-311.

86. Sun I-C, Eun D-K, Koo H, et al. Tumor-targeting gold particles for dual computed tomography/optical cancer imaging. Angew. Chem. Int. Ed. Engl. $2011 ; 50: 9348-9351$.

87. Jones BL, Manohar N, Reynoso F, et al. Experimental demonstration of benchtop x-ray fluorescence computed tomography (XFCT) of gold nanoparticleloaded objects using lead- and tin-filtered polychromatic cone-beams. Phys. Med. Biol. 2012;57:N457-67.

88. Kuang $Y$, Pratx G, Bazalova $M$, et al. First demonstration of multiplexed X-ray fluorescence computed tomography (XFCT) imaging. IEEE Trans Med Imaging. 2013;32:262-267.

89. Lawrence YR, Vikram B, Dignam JJ, et al. NCI-RTOG translational program strategic guidelines for the early-stage development of radiosensitizers. J. Natl. Cancer Inst. 2013;105:11-24.

90. Zheng Y, Sanche L. Gold nanoparticles enhance DNA damage induced by anti-cancer drugs and radiation. Radiation Research. 2009;172:114-119. 
Table 1: Pathway to implementing GNPs as clinical agents.

\begin{tabular}{|c|c|}
\hline Stage & Aims \\
\hline $\begin{array}{l}\text { Tumor site } \\
\text { identification }\end{array}$ & $\begin{array}{l}\text { Identify a tumor site, which may benefit significantly } \\
\text { from introduction of GNP contrast agents (see table 2). }\end{array}$ \\
\hline GNP design & $\begin{array}{c}\text { Design a GNP to efficiently target tumor site, } \\
\text { optimizing particle size and functionalization based on } \\
\text { tumor characteristics (table } 3 \text { ). }\end{array}$ \\
\hline in vitro validation & $\begin{array}{l}\text { in vitro models can be used to confirm basic } \\
\text { biocompatibility of GNP preparation and } \\
\text { radiosensitizing properties (table 4). }\end{array}$ \\
\hline in vivo validation & $\begin{array}{l}\text { Validated GNP preparations should undergo in vivo } \\
\text { validation in preclinical models. This should include } \\
\text { both, toxicity testing in relevant animal models, as well } \\
\text { as validation of radiosensitizing effects in models } \\
\text { recapitulating relevant tumor biology (table } 4 \text { ). }\end{array}$ \\
\hline Physics validation & $\begin{array}{l}\text { Physics models must be tested and updated as } \\
\text { necessary to enable accurate planning and dosimetry } \\
\text { in situations where GNPs introduce significant } \\
\text { concentrations of high-Z materials within treatment } \\
\text { fields. }\end{array}$ \\
\hline Clinical validation & $\begin{array}{c}\text { Following comprehensive pre-clinical validation, GNPs } \\
\text { must be validated through the established clinical trial } \\
\text { pathway, ensuring acceptable toxicity, compatibility } \\
\text { with existing chemotherapeutic regimes and } \\
\text { radiobiological effectiveness (table 5). }\end{array}$ \\
\hline
\end{tabular}


Table 2: Characteristics of tumor sites suitable for GNP-enhanced radiation therapy

\begin{tabular}{|c|c|}
\hline Characteristic & Rationale \\
\hline $\begin{array}{c}\text { Existing radiotherapy } \\
\text { treatment site }\end{array}$ & $\begin{array}{c}\text { Enhancing existing radiotherapy treatment } \\
\text { provides the most rapid and efficient pathway } \\
\text { to develop and deploy a new contrast agent }\end{array}$ \\
\hline $\begin{array}{c}\text { Clinical benefit for dose } \\
\text { escalation }\end{array}$ & $\begin{array}{c}\text { Sites with proven benefit of conventional dose } \\
\text { escalation are expected to benefit from GNP } \\
\text { dose escalation. }\end{array}$ \\
\hline $\begin{array}{c}\text { Limits to conventional dose } \\
\text { escalation due to normal } \\
\text { tissue }\end{array}$ & $\begin{array}{c}\text { Targeted GNP dose escalation offers greatest } \\
\text { benefit in sparing nearby sensitive organs }\end{array}$ \\
\hline $\begin{array}{c}\text { Tumors with characteristic } \\
\text { markers or structures }\end{array}$ & $\begin{array}{c}\text { GNPs designed to target tumor-specific } \\
\text { characteristics offer greater specificity than } \\
\text { passively targeted approaches. }\end{array}$ \\
\hline
\end{tabular}


Table 3: Important characteristics of GNPs for treatment enhancement

\begin{tabular}{|c|c|}
\hline Characteristic & Rationale \\
\hline High tumor specificity & $\begin{array}{c}\text { High GNP tumor specificity offers improved } \\
\text { contrast between healthy organs and tumor } \\
\text { volume, as well as reducing the total amount of } \\
\text { GNPs required during treatment }\end{array}$ \\
\hline Effective biodistribution & $\begin{array}{c}\text { GNPs must be capable of reaching the tumor } \\
\text { volume and be taken up without accumulating } \\
\text { in other organs or being cleared through RES. }\end{array}$ \\
\hline Persistent uptake in tumors & $\begin{array}{c}\text { Long GNP half-lives reduce the number of } \\
\text { GNP treatments required during therapy, } \\
\text { reducing logistical and financial burden. }\end{array}$ \\
\hline Compatibility with & $\begin{array}{c}\text { Negative interactions with other agents may } \\
\text { counteract benefit of GNP-enhanced therapy }\end{array}$ \\
\hline In vivo imaging potential & $\begin{array}{c}\text { Effective imaging techniques can provide } \\
\text { useful diagnostic information about the } \\
\text { patient's disease, as well as inform predictive } \\
\text { models of treatment outcome. }\end{array}$ \\
\hline Radiosensitizing potential & $\begin{array}{c}\text { All of the above factors must be balanced with } \\
\text { the GNPs' radiosensitizing properties, ensuring } \\
\text { that functionalization for uptake or specificity } \\
\text { does not interfere with core sensitizing } \\
\text { properties. }\end{array}$ \\
\hline
\end{tabular}


Table 4: Pre-clinical experimental results needed to inform development of GNPbased therapies

\begin{tabular}{|c|l|l|}
\hline \multicolumn{2}{|c|}{ Experimental Validation } & \multicolumn{1}{c|}{ Key Data } \\
\hline \multirow{3}{*}{ In vitro } & biocompatibility & $\begin{array}{l}\text { GNP preparations and concentrations } \\
\text { which are tolerated by representative } \\
\text { normal tissues }\end{array}$ \\
\cline { 2 - 3 } & radiosensitization & $\begin{array}{l}\text { Preliminary quantification of relationship } \\
\text { between GNP delivery and } \\
\text { radiosensitization to inform pre-clinical and } \\
\text { clinical experiments }\end{array}$ \\
\hline \multirow{4}{*}{ In vivo } & biocompatibility & $\begin{array}{l}\text { Confirmation that particles are non-toxic in } \\
\text { concentrations intended for clinical use, } \\
\text { and that these particles or coatings do not } \\
\text { have unforeseen side effects. }\end{array}$ \\
\cline { 2 - 3 } & radiosensitization & $\begin{array}{l}\text { Quantification of GNP radiosensitization in } \\
\text { models which as closely as possible } \\
\text { match clinical setting to confirm efficacy } \\
\text { and inform clinical dosing and treatment } \\
\text { planning. }\end{array}$ \\
\hline
\end{tabular}


Table 5: Clinical experimental results required for final validation of GNPenhanced radiotherapy

\begin{tabular}{|c|l|}
\hline Clinical Validation & \multicolumn{1}{c|}{ Key Data } \\
\hline Physics Validation & $\begin{array}{l}\text { Confirmation that anticipated GNP uptake and } \\
\text { distribution will not significantly impact on } \\
\text { radiotherapy planning procedures, or } \\
\text { developing appropriate techniques for planning } \\
\text { GNP-enhanced therapies. }\end{array}$ \\
\hline $\begin{array}{c}\text { Human biodistribution \& toxicity } \\
\text { (Phase 0/1) }\end{array}$ & $\begin{array}{l}\text { Information on biodistribution and potential } \\
\text { toxicity of GNPs in humans, as well as } \\
\text { clinically feasible dosing and interactions with } \\
\text { other agents (e.g. chemotherapeutics) and } \\
\text { quantification of retention of GNPs inside the } \\
\text { tumor }\end{array}$ \\
\hline Human efficacy & $\begin{array}{l}\text { Validation and quantification of } \\
\text { radiosensitization in patient treatment, } \\
\text { improvements in tumor control and overall } \\
\text { survival }\end{array}$ \\
\hline
\end{tabular}




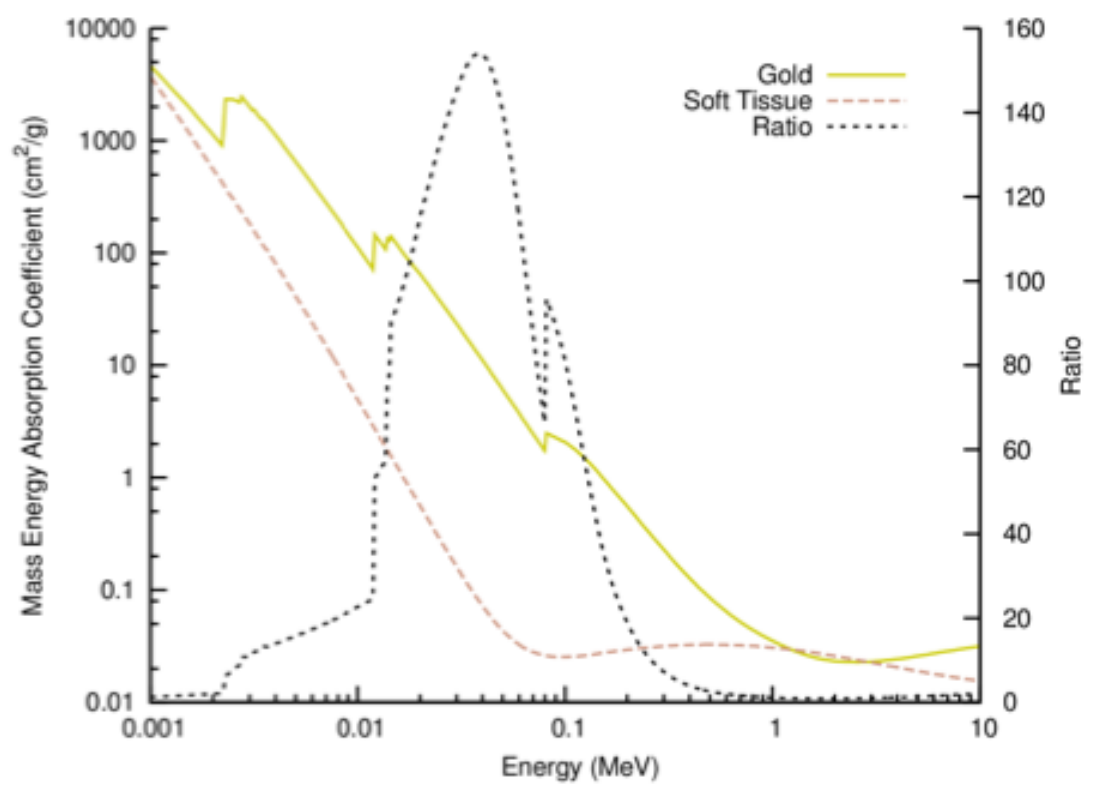

Figure 1: Mass energy absorption for gold and soft tissue and the ratio. Figure with permission from (3). 

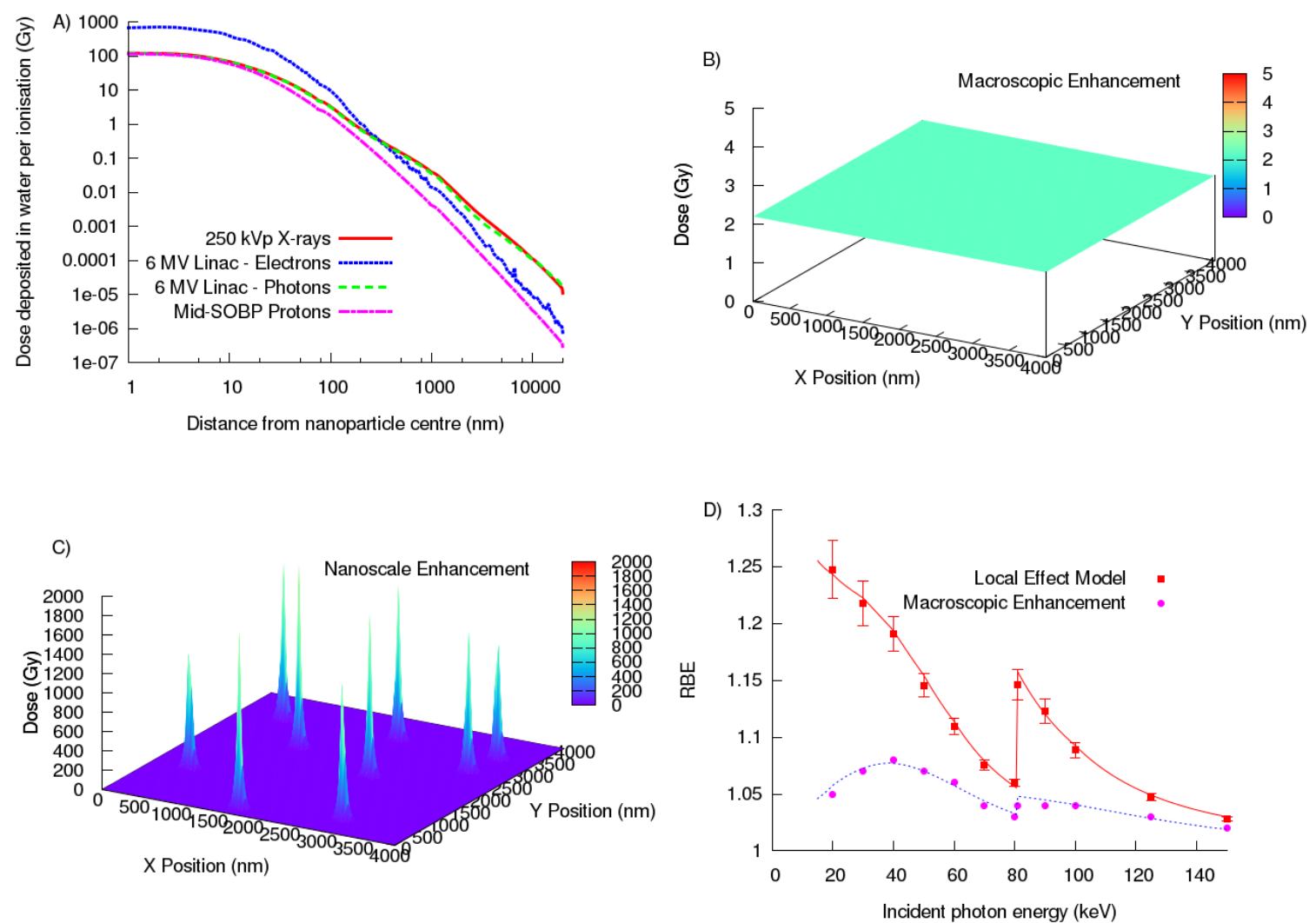

Figure 2: Illustration of the radiosensitization effect of GNPs: A) Comparison of the radial dose distribution per ionization as obtained from Monte Carlo simulations performed with TOPAS (16) for a single $50 \mathrm{~nm}$ GNP at the center of a proton spread out Bragg peak (SOBP), for a $250 \mathrm{kVp}$ photon field at $1 \mathrm{~mm}$ depth in water and for the electron and photon components of a Varian $6 \mathrm{MV}$ field at the $80 \%$ depth dose position, B) a $10 \%$ dose enhancement calculated for the addition of GNPs to a uniform 2 Gy by $50 \mathrm{keV} X$-ray irradiation if the additional dose is calculated with macroscopic methods vs. C) the dose enhancement when considering doses at the microscopic scale around individual GNPs, and $D$ ) the resulting RBE caused by the addition of GNPs comparing macroscopic and microscopic (based on an approach following the Local Effect Model) biological modeling for keV X-ray irradiation. 

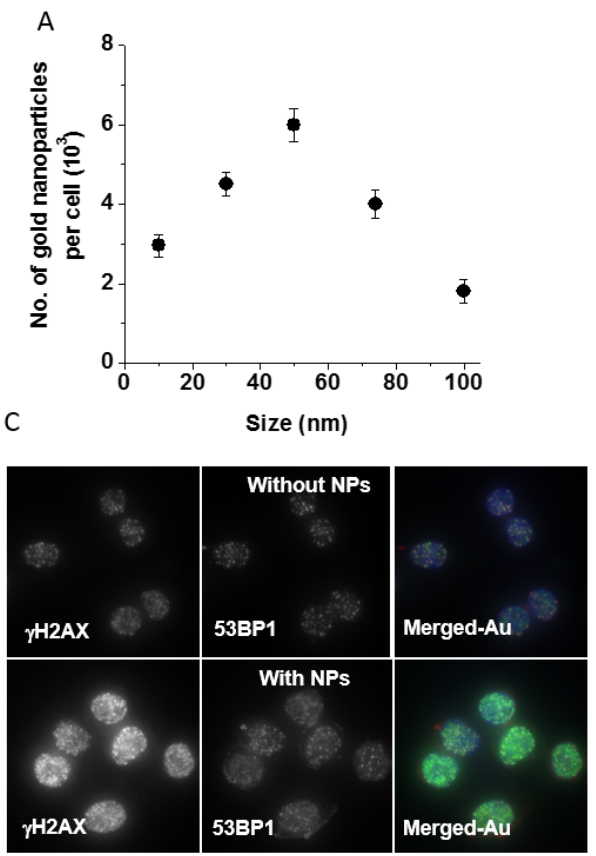

B

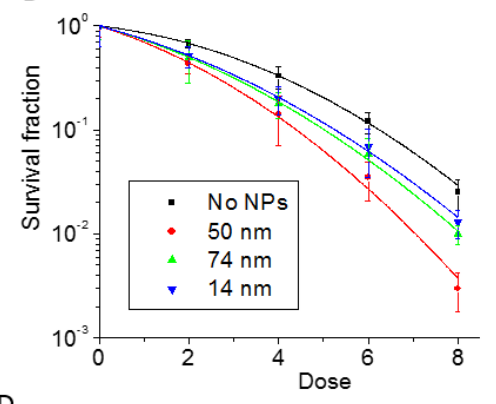

D

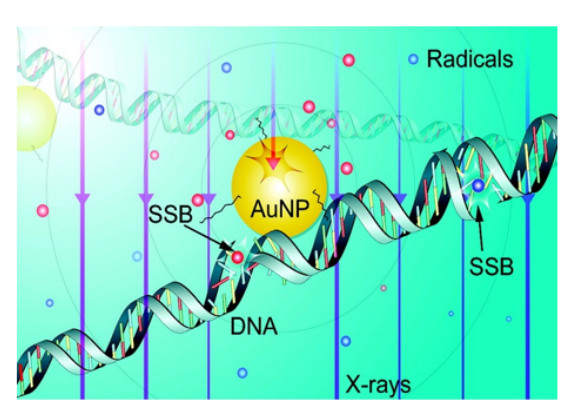

Figure 3: Size dependence of GNP uptake and sensitization. A. Cellular uptake of GNPs with a diameter size of 10-100 nm. B. Cell survival curves based on a clonogenic assay for the same cells as in A. C. Cells that internalized GNPs showed higher DNA double strand breaks after radiation compared to the control cells with no internalized GNPs. D. Schematic showing enhanced free radial formation during the radiation treatment in the presence of $\operatorname{GNPs}(18,19,22)$. 

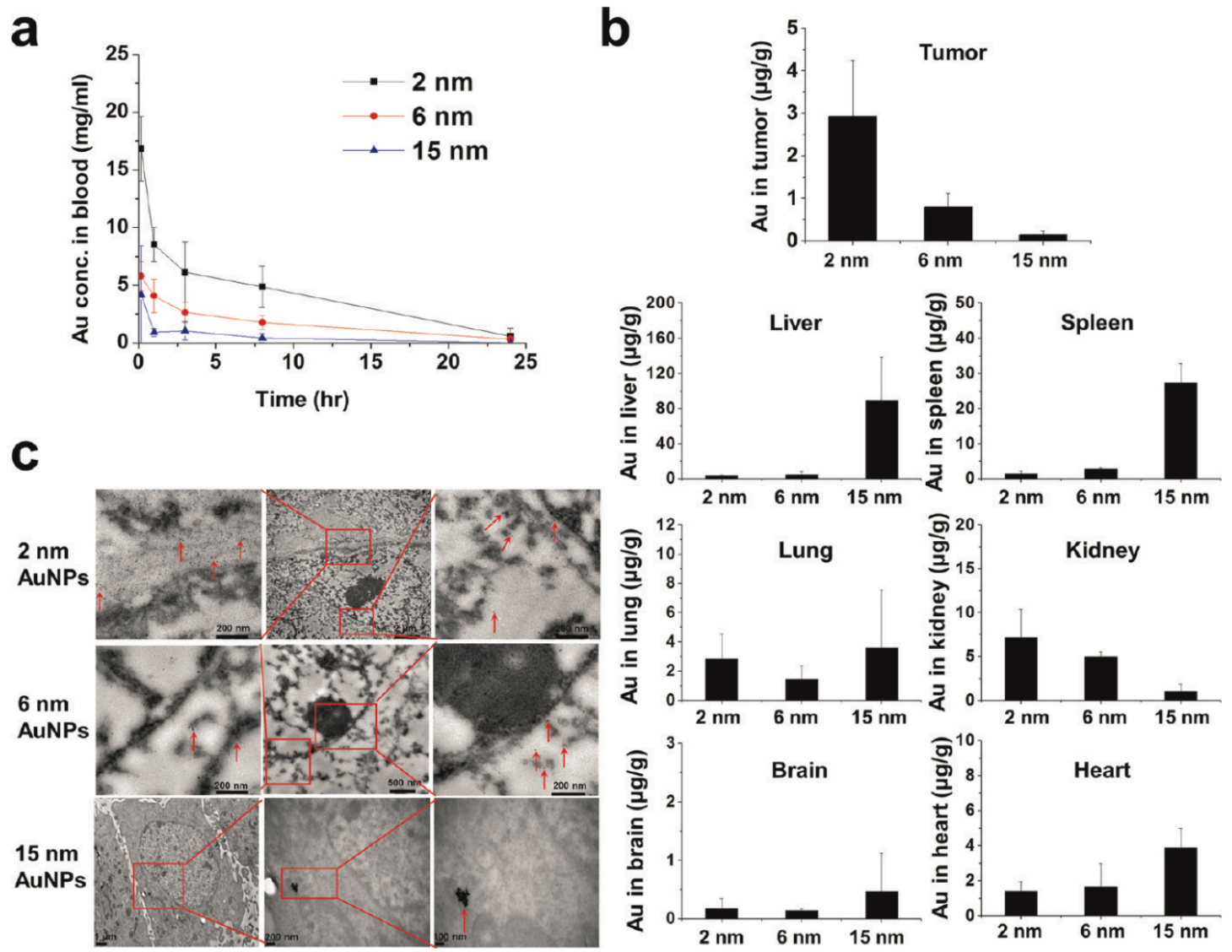

Figure 4: Pharmacokinetics and biodistribution of 2, 6, and $15 \mathrm{~nm}$ GNPs coated with tiopronin. A) Blood elimination profiles of gold following a single intravenous injection of GNPs at a dose of $5 \mathrm{mg}$ gold/kg in tumor-bearing mice. Data represent mean $\pm S D(n=3)$. B) Gold content in tumor, heart, liver, spleen, lung, brain, and kidney $24 \mathrm{~h}$ after intravenous injection of GNPs at $5 \mathrm{mg}$ gold $/ \mathrm{kg}$. Data represent mean $\pm S D(n=3)$ C) Representative TEM micrographs of tumor tissue taken $24 \mathrm{~h}$ after the administration of GNPs. Figure with permission from (33). 

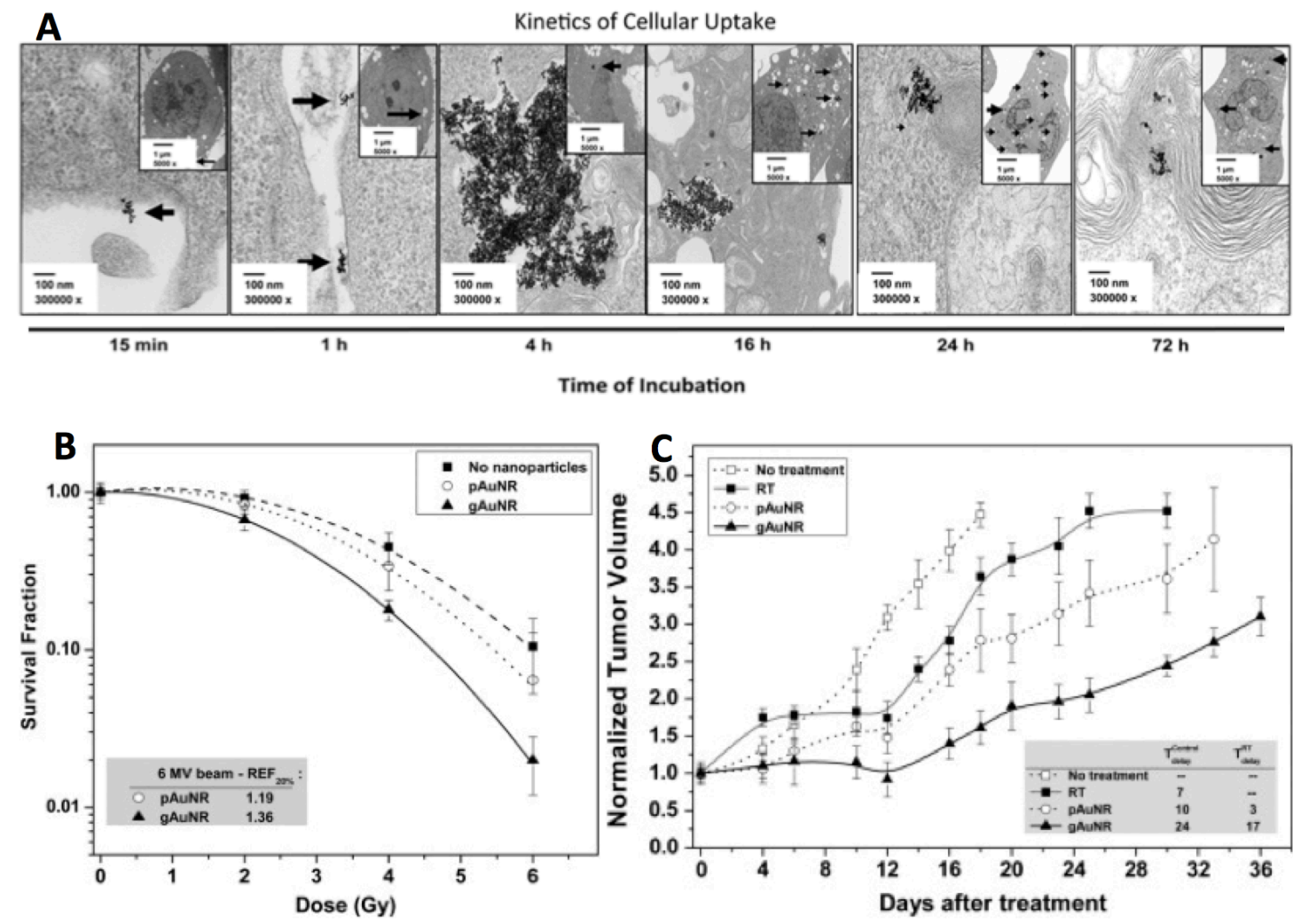

Figure 5: Radiosensitization by conjugated GNPs injected intravenously, irradiated with a bolus laid over each mouse. A) TEM images of gold nanorods (AuNRs) conjugated to goserelin, a gonadotropin releasing hormone agonist, demonstrating binding to the cell surface of prostate cancer cells and internalization over time to endocytotic vesicles. B) Clonogenic survival curves demonstrating greater radiosensitization with goserelin-conjugated AuNRs (gAuNRs) than PEGylated AuNRs (pAuNRs) when irradiated with 6MV photons. C) Tumor growth curves of prostate cancer xenografts treated with single dose $6 M V$ radiation in the presence of AuNRs demonstrating greater delay in tumor regrowth when treated with gAuNRs than pAuNRs. Reprinted with permission from reference 6 . 


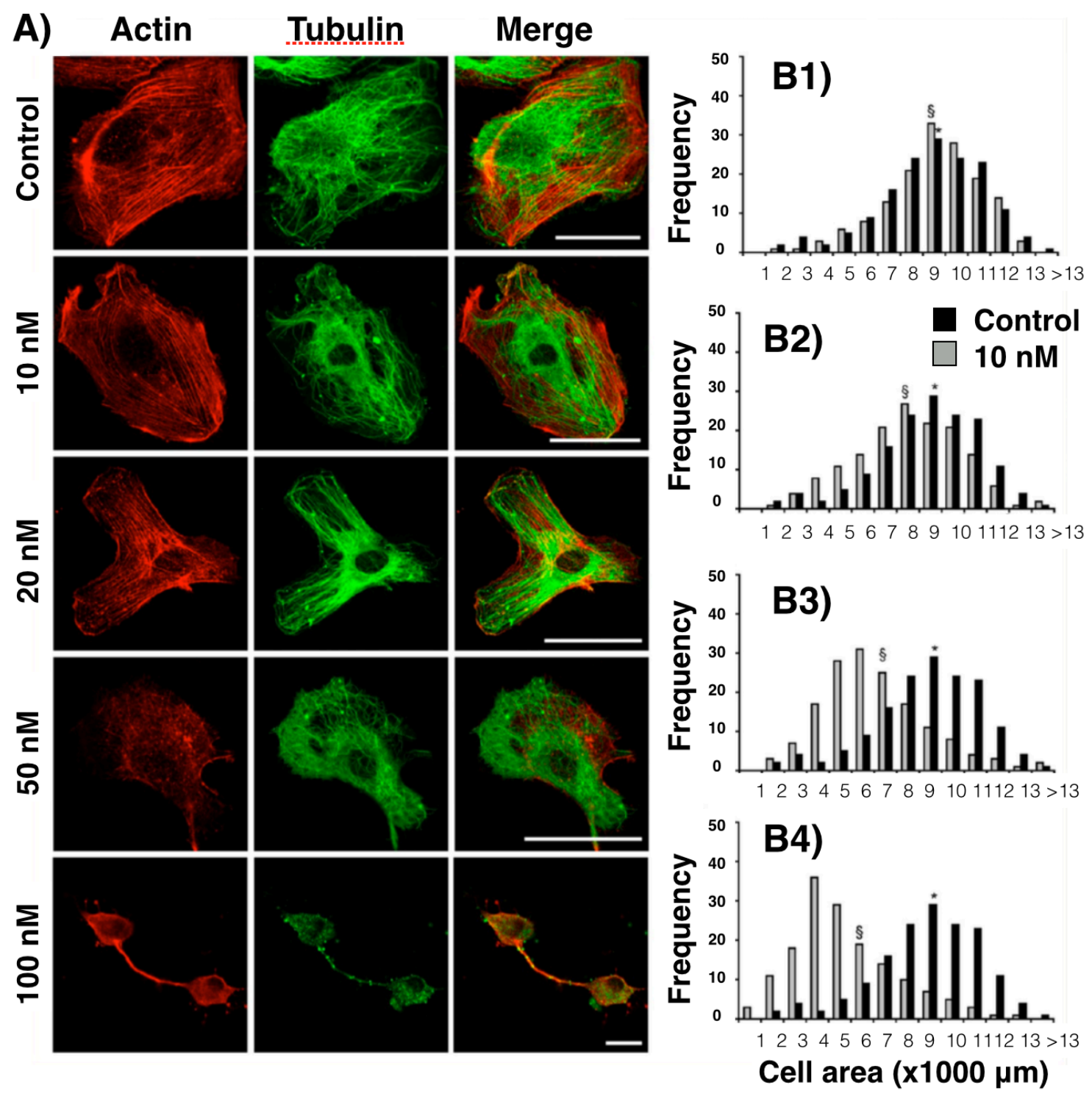

Figure 6: A) Representative confocal images of control HUVECs or cells exposed to $10,20,50$, or $100 \mathrm{nM}$ GNP for $24 \mathrm{~h}$ at 1 day post-NP-labeling. The left column depicts actin staining (red), the middle column depicts $\alpha$-tubulin (green), and the right column is a merged image of both actin and $\alpha$-tubulin. Scale bars: $50 \mu \mathrm{m}$. (B) Histograms representing the cell areas of control cells (dark gray) and cells incubated for $24 \mathrm{~h}$ with GNPs at $10 \mathrm{nM}$ (B1), $20 \mathrm{nM}$ (B2), $50 \mathrm{nM}$ (B3), and 100 $n M(B 4)$. The average cell area is indicated with (*) for control cells and with (\$) for NP-treated cells. Figure with permission from (65). 

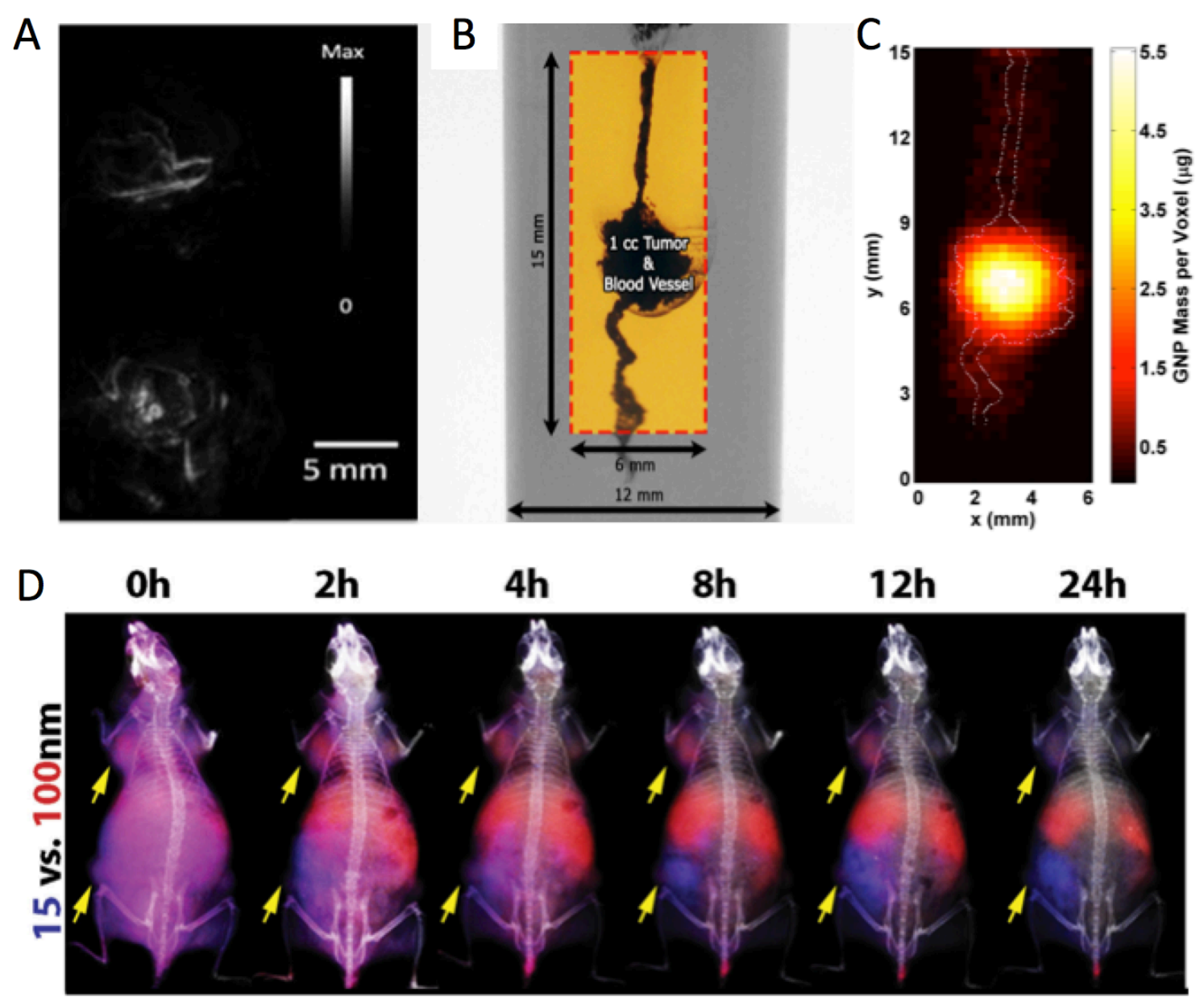

Figure 7: A: Photoacoustic images of tumors in vivo before (top) and $6 \mathrm{~h}$ after (bottom) intravenous administration of gold nanorods documenting extravasation of nanoparticles from vasculature to tumor parenchyma. Reproduced with permission from reference (69). B: XFCT imaging phantom with simulated GNPladen blood vessel and $\sim 1 \mathrm{~cm}^{3}$ tumor. The box shows the region of interest that was imaged. C: 2-D x-ray fluorescence image of the region of interest within the phantom. The GNP distribution within the simulated tumor region and blood vessels is shown, with the maximum value of $\sim 5.5 \mu \mathrm{g}$. The outline of the irradiated GNP-containing structures is overlaid on the fluorescence image, showing a good match between the images around the tumor region. From Ref. 74 with permission (pending). D. Fluorescence image guided biosdistribution studies with GNPs of MDA-MB-435 tumored (yellow arrows) mice injected simultaneously with $15 \mathrm{~nm}$ (blue fluorescence) and $100 \mathrm{~nm}$ (red fluorescence) gold nanoparticles which were coated with PEG $5 \mathrm{kDa}$, and fluorescently labelled with Kodak X-Sight 670 and Alexa Fluor 750, respectively (33) (Permission pending). 